\title{
WELFARE E SINDACATO TRA AZIENDA E TERRITORIO: \\ INTERVISTA A TIZIANA BASSO - CGIL VENETO
}

\author{
di Alberto Mattei*
}

Giovedì 4 luglio 2019, presso Ires Veneto, si è svolta la seguente intervista con Tiziana Basso, segretaria confederale di Cgil Veneto (d'ora in avanti nell'intervista: TB). Il tema è il welfare aziendale: inquadramento del tema a partire dal lessico, il rapporto con il Welfare State e gli sviluppi del welfare locale territoriale (a cura di Alberto Mattei, d'ora in avanti nell'intervista: $A M$ ).

ALBERTO MATTEI - Innanzitutto, il lettore di economia e società regionale chi ha di fronte?

TIZIANA BASSO - Sono componente della segreteria Cgil del Veneto. Come segretaria ho alcune deleghe, tra cui la contrattazione, che per me rappresenta soprattutto quella di secondo livello, anche se, naturalmente come confederazione, siamo coinvolti a livello nazionale per le politiche contrattuali più generali. Ho anche la delega per il settore dell'artigianato, dove da molto tempo, in Veneto, si sono sviluppate relazioni e anche una contrattazione che ha dato vita ad un'esperienza molto avanzata di welfare contrattuale. Il welfare nella bilateralità artigiana, però, ha caratteristiche peculiari. Rispetto al tema del welfare aziendale, come Cgil del Veneto, da tempo, abbiamo aperto una discussione. La discussione non ha riguardato solo chi si occupa di contrattazione ma tutta l'organizzazione, visti i riflessi sul welfare pubblico, in linea con il percorso avviato anche a livello nazionale.

AM - Dal punto di vista del lessico, partiamo da "welfare aziendale": dal lato di chi opera quotidianamente a livello sindacale, cosa si intende con questa espressione?

* Dipartimento di Scienze Giuridiche, Università degli Studi di Verona.

ECONOMIA E SOCIETÀ REGIONALE - ISSN 1827-2479 - XXXVII(2) 2019 - INNOVAZIONE SOCIALE E DOI: $10.3280 / E S 2019-002010$

NUOVO WELFARE TERRITORIALE 
TB - Si tratta, per noi, come organizzazione sindacale, di un tema affrontato fin da subito. Come Cgil del Veneto siamo stati tra i primi a sviluppare un lavoro di approfondimento. Devo dire che in tutte le scelte politiche fatte da Cgil, a partire dal Protocollo con Cisl e Uil sul modello di contrattazione inviato a tutte le controparti, sono state definite con precisione le linee guida su cosa si intende per welfare contrattuale. In questo senso, per noi, il tema del welfare aziendale è da leggere come declinazione del welfare contrattuale.

Noi abbiamo tre filoni che riteniamo principali su cui intervenire con la contrattazione: in primo luogo, la previdenza integrativa; in secondo luogo, la sanità integrativa, su cui c'è una forte pressione a livello interno rispetto alla congruità degli interventi e anche in merito alla relazione con la sanità pubblica, su cui tornerò più avanti nel corso dell'intervista; e, in terzo luogo, la conciliazione tra tempi di vita e tempi di lavoro, con intrecci tra formazione e riqualificazione professionale e tutte quelle che possono essere le iniziative di condivisione tra il tempo di vita e di lavoro.

La norma fiscale riconosce vantaggi anche ad altre misure ma, per noi, come sindacato, si tratta di flexible benefits e non di welfare contrattuale o aziendale. Purtroppo, le Piattaforme in circolazione che offrono alle aziende servizi di così detto welfare, propongono anche flexible benefits ed è complicato nella contrattazione, per il sindacato, vincolare la scelta del lavoratore.

AM - Tu quindi distingueresti tra welfare contrattuale e flexible benefits?

TB - Sì, per noi è chiara la distinzione tra welfare e benefit. Il welfare contrattuale può avere un effetto positivo quando ha anche una valenza sociale. E questo vale sia che si tratti di trasformazione del premio in welfare che si tratti invece di welfare aggiuntivo. Abbiamo sempre tentato di bloccare quella che è la trasformazione e lo scambio tra salario e beni. Per esempio, un buono Amazon o un buono benzina al posto del salario non hanno alcun effetto sociale positivo: si tratta di uno scambio che per noi è da evitare. Considero comunque, nel concreto, che all'interno della contrattazione aziendale ci siano delle mediazioni possibili, ma per noi l'obiettivo è quello di dare una valenza sociale al welfare aziendale. Anche perché è finanziato dalla fiscalità generale.

AM - Tuttavia, a mio avviso, non è sempre così immediata la distinzione tra ciò che ha una valenza sociale e ciò che è scambio tra meno salario e più welfare, specialmente quando quest'ultimo consiste, per esempio, in versamento alla previdenza integrativa. Possono essere previste sovrapposizioni? 
TB - Va considerato che a monte c'è un'agevolazione incentivata e fortemente sostenuta dal legislatore, come una vera e propria spinta verso la trasformazione dei premi. Di conseguenza, c'è stato un impulso verso questa scelta a favore del welfare aziendale. Se però guardiamo i dati, i lavoratori scelgono ancora in gran parte il salario. Così vale per i dati del Ministero $^{1}$, così anche per la nostra Regione: la scelta individuale è declinata sul salario. Questo deve far riflettere (anche il legislatore): c'è, per i lavoratori, una forte esigenza salariale che non trova risposta nella spinta verso altri bisogni.

AM - All'opposto, la scelta di optare per il salario in favore del welfare può portare ad una questione di mancata contribuzione?

TB - È uno dei temi su cui si è concentrata la nostra riflessione. Assieme alla riduzione delle risorse raccolte e destinate al Welfare State, riteniamo possa essere un problema concreto. Per tale motivo, è necessario dare un'informazione adeguata ai lavoratori, e noi ci siamo impegnati in una campagna di formazione anche dei nostri delegati. La mancata contribuzione può incidere anche in maniera significativa: è stato un punto valutato attentamente dai lavoratori informati nel fare la scelta. Anche in una visione meramente individuale e utilitaristica, il conto totale del beneficio si deve fare con tutti gli elementi.

AM - E sulla sanità integrativa?

TB - A livello nazionale e qui in Veneto, stiamo ragionando su alcuni elementi. A partire dalla fine degli anni Novanta, la norma ha previsto la possibilità di istituire attraverso la contrattazione collettiva fondi sanitari integrativi. L'esperienza più vicina a noi è quella del Fondo sanitario per l'artigianato veneto nato nel 2013.

In questi anni, abbiamo sviluppato, anche sulla base di questa esperienza, due valutazioni prioritarie: la sanità deve essere veramente integrativa e non sostitutiva. Questo implica che le parti che governano il Fondo sanitario devono contrattare il nomenclatore delle prestazioni che vengono riconosciute e non lasciare la scelta alle assicurazioni. Solo in questo modo avremo prestazioni integrative e congrue con i bisogni del settore. Ad

1 Ad esempio, i Report di aggiornamento pubblicati periodicamente dal Ministero del Lavoro e delle Politiche Sociali riguardanti le procedure di deposito a livello telematico dei contratti aziendali e territoriali sulla detassazione dei premi di produttività ai sensi del decreto interministeriale del 25 marzo 2016. Si segnala anche il Primo Rapporto sulla contrattazione di secondo livello promosso da Cgil Nazionale e Fondazione Giuseppe Di Vittorio. 
esempio, nel Fondo per l'artigianato abbiamo deciso di dedicare risorse per la prevenzione tenendo conto dell'aumento dell'età degli addetti.

L'altra valutazione è la salvaguardia del Ssn pubblico e universale. Quindi convenzionamento dei fondi con il sistema pubblico (al posto del privato). In questo modo le risorse che le aziende versano e che, ricordiamocelo, godono di agevolazioni fiscali, rientrano nel sistema pubblico portando beneficio a tutta la collettività. La sanità integrativa è welfare lavorativo, riguarda chi lavora, se la indirizziamo verso il pubblico va a vantaggio anche degli esclusi perché migliora le liste d'attesa, porta risorse fresche per aumentare le prestazioni e, quindi, si può tradurre in un sostegno al territorio. È un tema complesso, per cui è necessario ragionare con le singole Regioni, considerando che comunque i fondi sono nazionali e vengono poi declinati a livello regionale. Sul piano concreto, con il meccanismo di convenzionamento ci sarebbe un'ulteriore incentivazione per accedere al sistema pubblico, che già i lavoratori veneti privilegiano. Un modo concreto per contrastare i dualismi che il welfare aziendale può generare.

$\mathbf{A M}-C^{\prime}$ è un dibattito interno a Cgil Nazionale per cui parlare di welfare aziendale vuol dire mettere in crisi il sistema di Welfare State. Alla luce della tua esperienza sul campo, cosa ne pensi?

TB - È aperta sicuramente una discussione interna, anche alla luce dei primi risultati della contrattazione. Il welfare aziendale esiste da tanto tempo, nel 2015 ha solo trovato un nuovo impulso legislativo. Il tema di fondo è che la detassazione rischia di svuotare le entrate tributarie andando ulteriormente a tagliare risorse, per esempio, nella Sanità. Si dice che il welfare aziendale nasce per far fronte alla crisi del sistema di welfare pubblico. Ciò, però, non deve significare che, a fronte della spinta al welfare aziendale che viene incentivato fiscalmente dalla normativa, si debba andare a compromettere ulteriormente il Welfare State. Il welfare aziendale deve portare benefici a tutti, perché è finanziato dalla fiscalità generale. Non si demonizza lo strumento, ma è importante capire come usarlo bene, finalizzarlo correttamente e avere chiaro che, per esempio, mettere a disposizione dei dipendenti l'uso della palestra non è fare welfare aziendale. Se l'azienda vuole offrite la palestra ai propri lavoratori, lo faccia con le proprie risorse, non con quelle dalla collettività!

AM - Parlare di welfare aziendale vuol dire pertanto parlare di un welfare che non abbatte il sistema pubblico?

TB - Quando parliamo di contrattazione inclusiva, non parliamo solo di contrattazione collettiva di secondo livello che coinvolga tutti i lavoratori presenti in un determinato sito, indipendentemente dalla tipologia contrat- 
tuale applicata, ma anche di un welfare aziendale che incida su un territorio, con l'obbiettivo di includere anziché escludere. Il welfare aziendale rischia, se non ben vigilato, di avere una valenza esclusiva, nel senso di ridursi a un welfare riservato ai pochi che lavorano in quell'azienda escludendo tutti gli altri. Dobbiamo trovare le modalità contrattuali che consentano il coinvolgimento e il trasferimento dei benefici a più soggetti. Si tratta, anche per noi, di un percorso che richiede passaggi culturali da compiere.

AM - Questa valenza di territorio che hai posto entra in un altro fronte: quali sono le azioni per portare il welfare dall'azienda alla comunità entro cui l'impresa opera?

TB - Ci sono cose che entrano nell'abicì sindacale, che ci vengono trasmesse a partire dai corsi per Rsu. Quando si discute di asilo aziendale è scontato, per noi, che tale asilo si debba aprire al territorio in cui opera l'azienda e sia messo a disposizione del territorio. Stiamo in questo momento però ragionando di altro. L'esperienza fatta, per esempio con alcuni Comuni della Provincia di Padova, in cui si è ragionato di quali benefici potessero venire dalla contrattazione di secondo livello per il territorio, ha prodotto un'esperienza positiva. È stato sottoscritto un protocollo con i Comuni e tutte le Associazioni sindacali e datoriali nel quale si è convenuto che la contrattazione aziendale, che poneva tra le azioni di condivisione il rimborso per le spese del nido, avrebbe potuto essere attuata attraverso il convenzionamento con l'asilo nido del Comune. Quest'ultimo ha modificato il proprio Regolamento comunale consentendo l'accesso al nido non solo ai residenti, ma anche a chi lavora nel territorio. In tal modo, l'azienda rimborsa una parte della retta e, al tempo stesso, l'aumento dell'utenza ha consentito di conservare il servizio del nido che, altrimenti, in quel territorio sarebbe stato chiuso. Abbiamo fatto rientrare, così, le risorse del welfare aziendale nel territorio in un'esperienza di concertazione tra pubblico e privato, con Associazioni sindacali e datoriali, generando positività per tutti.

La stessa cosa andrebbe fatta per la previdenza integrativa che deve intervenire sull'economia reale, come dichiariamo da anni. In questi mesi, sono in corso discussioni con Cassa Depositi e Prestiti per dare le giuste garanzie ai lavoratori. Gli investimenti potrebbero essere anche caratterizzati da interventi che rispondano a esigenze sociali e territoriali. Mi chiedo: perché le risorse della previdenza non possono essere investite, per esempio, nelle residenze per anziani?

Così anche per la formazione professionale pagata dall'azienda, che può essere aperta a costi agevolati a chi abita nel territorio o, ancora, per la sa- 
nità integrativa messa a disposizione del territorio che interviene sugli extra Lea (come ha scelto di fare, per esempio, la Regione EmiliaRomagna $\left.{ }^{2}\right)$.

AM - Un intervento diretto nella sanità integrativa da parte dell'ente pubblico?

TB - L'esempio che voglio fare è quello di un fondo integrativo che offra l'assistenza domiciliare per il dipendente. Si potrebbe pensare ad una sinergia tra il Comune, che deve garantire l'assistenza ai propri cittadini, e il fondo.

AM - Quindi, in tal caso, sarebbe il sistema pubblico che va ad integrare il privato, avendo una ricaduta sul territorio?

TB - Esatto. Si tratterebbe di un'evoluzione complessa. Teniamo conto che i fondi funzionano all'interno di una cornice solidaristica. Funzionano quando ci sono molte persone che fanno una cassa comune, altrimenti, se cioè vi aderiscono poche persone, c'è il rischio che non siano sostenibili.

AM - Tornando alle misure più tradizionali di benefit, a tuo avviso il welfare contrattuale può essere letto come un meccanismo di fidelizzazione?

TB - Nasce proprio così. Penso ad aziende come Marzotto o Lanerossi, dove c'erano benefici a favore del personale dipendente, quali gli asili nido, le visite mediche gratuite per i familiari, le scuole, ecc., che consentivano di creare benessere. Poi c'è stato il welfare in azienda (penso alla nostra regione) che si è manifestato nei buoni libri scolasti, nei rimborsi delle spese mediche dei famigliari, nel carrello della spesa (che non è welfare) ecc., cioè si dava al lavoratore qualcosa in più che lo facesse "stare bene", così da generare un effetto positivo sulla produttività.

2 Si veda il Patto per il Lavoro. Un nuovo sviluppo per una nuova coesione sociale sottoscritto da Regione Emilia-Romagna e numerosi stakeholders, tra cui OO.SS., nel 2015. All'interno del testo si prevede: una «sperimentazione per l'istituzione di un fondo regionale per la sanità integrativa per l'erogazione di prestazioni extra Lea». Tale fondo verrebbe «alimentato dalla contrattazione nazionale, articolata, e da risorse aggiuntive derivanti dall'adesione di cittadini anche non lavoratori. Confermando comunque che il riferimento anche per adesioni di carattere contrattuale va inteso in termini di volontarietà. Le risorse saranno collegate al Ssr attraverso meccanismi di convenzionamento» (Regione EmiliaRomagna, 2015: 23). Un'altra esperienza peculiare è quella di Sanifonds - Fondo Sanitario Integrativo della Provincia autonoma di Trentino, i cui soci sono l'ente pubblico e numerosi stakeholders, tra cui OO.SS., che garantisce agli iscritti prestazioni sanitarie integrative del sistema sanitario pubblico a favore di dipendenti pubblici e privati. 
Con la legislazione del 2015, il tutto si è rovesciato, a partire dalla produttività verso il benessere: se aumento la produttività ho il premio che mi consente di accedere a prestazioni di welfare. In una battuta, io posso stare bene solo se aumento la produttività o l'efficienza. In realtà, dai lavoratori questo non è stato visto come qualcosa in più, come avveniva prima, ma è stato visto piuttosto come il contrario.

L'opzione del welfare al posto del premio in denaro è ancora minoritaria, e infatti cominciano a saltare le prime Piattaforme di servizi, perché il mercato è limitato.

$\mathbf{A M}-$ E per il territorio regionale?

TB - Per il territorio veneto, la contrattazione, sia confederale che di categoria, ha fatto del welfare collettivo nell'artigianato un'esperienza concreta. Sono state attuate modalità inclusive e anche qui ci si sta interrogando su come migliorare la relazione con il territorio. In prospettiva, è da sperimentare un'attività di intreccio, tornando al territorio in modo da dare una maggior inclusività. Penso, ad esempio, alla relazione con i Comuni per l'assistenza agli anziani. L'ulteriore differenza sta nel fatto che queste erogazioni non fruiscono dei benefici fiscali. Chiaramente, il welfare della bilateralità artigiana non è il welfare aziendale "tradizionale" che non è pensabile per la dimensione artigiana. L'intervento fiscale non ha prodotto un significativo aumento della contrattazione di secondo livello, come era nella volontà del legislatore.

AM - La legislazione avrebbe infatti come obiettivo anche lo sviluppo della contrattazione aziendale?

TB - L'obiettivo primario del welfare contrattato era quello di creare i presupposti per lo stare bene in azienda al fine di migliorare la produttività. Ora il paradigma si è rovesciato: si ha welfare solo se si aumenta la produzione. L'intervento fiscale non ha prodotto un aumento della contrattazione e, anzi, molto spesso ha ridotto la contrattazione solo al premio e all'eventuale trasformazione in welfare per fruire delle agevolazioni fiscali. In questo modo, temi a noi cari - come interventi sull'organizzazione del lavoro, salute e sicurezza, conciliazione dei tempi - sono rimasti marginali, riducendo anche la possibilità di aumentare concretamente la produttività.

Alla luce di questi risultati, pensiamo che sarebbe necessario e opportuno un ripensamento del legislatore sulle agevolazioni fiscali, perché, così come sono concepite attualmente, falliscono l'obiettivo di aumentare la contrattazione e di aumentare la produttività. 


\section{Riferimenti bibliografici}

Cgil Nazionale - Fondazione Giuseppe Di Vittorio (2019). Primo rapporto sulla contrattazione di secondo livello -- $<\mathrm{http}: / / \mathrm{www} . c g i l . i t / a d m i n \_n v 47 \mathrm{t} 8 \mathrm{~g} 34 / \mathrm{wp}-$ content/uploads/2019/01/Cgil-Fdv_Report-contrattazione-secondo-livello_11g en2019.pdf $>$.

Ministero del Lavoro e delle Politiche Sociali. Report detassazione -$<$ www.lavoro.gov.it $>$.

Regione Emilia-Romagna (2015). Patto per il Lavoro. Un nuovo sviluppo per una nuova coesione sociale. Bologna, 20 luglio -- $<\mathrm{http}$ ://www.regione.emiliaromagna.it/patto-per-il-lavoro/atti-e-documenti/patto_per_il_lavoro-1.pdf $>$.

Sanifonds Trentino. Fondo sanitario integrativo -- $<$ www.sanifonds.tn.it $>$. 